\title{
Empiema pleural por Streptococcus grupo anginosus en un preescolar y revisión de la literatura
}

PAOLA PIDAL M., JAVIERA BASAURE O.,

PRISCILLA PRADO D. y PEDRO ALARCÓN L.

\section{Streptococcus anginosus pleural emphyema in a child. Case report and review}

Left pneumonectomy in a child with congenital pulmonary malformation was performed two months prior to admission. Within one week he had high fever. Chest CT scan showed massive left pleural effusion. Both Streptococcus anginosus group and Prevotella spp. grew from the fluid obtained by pleural punction. Antimicrobial therapy was immediately initiated with clindamycin and penicillin. Streptococus anginosus group is quite confusing in terms of classification and taxonomy. Now it is well recognized that three species belong to this genera: $S$. anginosus $S$. constellatus and $S$. intermedius. They all are considered normal flora of the oropharynx, nasopharynx, gastrointestinal tract and vagina but also they have been identified as agents of suppurative infections in children and adults. Many reports show adequate response to penicillin, however there is some emerging resistance in strains isolated in other studies. In this article we also suggest some diagnostic and microbiologic recommendations.

Key words: Streptococcus anginosus group, Streptococcus milleri group, Streptococcus viridans group, Pleural effusion.

Palabras claves: Streptococcus grupo anginosus, Streptococcus milleri, Streptococcus grupo viridans, Empiema pleural.

\section{Caso clínico}

Paciente de sexo masculino, de 3 años 10 meses de edad, que ingresó al Hospital Padre Hurtado para someterse a neumonectomía izquierda por malformación pulmonar (probable síndrome de Williams Campbell). Se llegó a esta decisión después de dos años de seguimiento y una vez realizados los siguientes exámenes complementarios: TAC pulmonar que evidenciaba bronquiectasias saculares en gran parte del pulmón izquierdo, atelectasia parcial de este lóbulo y atelectasia del segmento basal posterior del lóbulo inferior izquierdo; fibrobroncoscopia que resultó normal y cintigrama de ventilación/perfusión que demostró una perfusión pulmonar izquierda igual a $4,3 \%$ y ventilación pulmonar izquierda igual a $6 \%$, interpretadas como severo compromiso difuso de la relación V/Q en pulmón izquierdo.
Con posterioridad a la neumonectomía evolucionó favorablemente siendo dado de alta al sexto día postoperatorio. Reingresó 43 días después de la cirugía, con historia de fiebre hasta $41^{\circ} \mathrm{C}$ durante una semana. Se le practicaron los siguientes exámenes: hemograma con 30.700 leucocitos/ $\mathrm{mm}^{3}$ (80\% granulocitos), PCR: $313 \mathrm{mg} / \mathrm{L}$, radiografía de tórax que demostró una extensa colección intratorácica izquierda (Figura 1), y una TAC pulmonar en la cual se observaba una gran colección intrapleural izquierda ocupando toda la cavidad y consolidación basal derecha (Figura 2). Se le realizó una punción pleural que dio salida a líquido turbio con $\mathrm{pH} 6,33$, glucosa $12 \mathrm{mg} \%$, leucocitos $4.400 / \mathrm{mm}^{3}$ (75\% de polimorfonucleares). Se inició tratamiento con cefotaxima más cloxacilina. De los cultivos en aerobiosis y anaerobiosis del líquido pleural se aislaron Streptococcus grupo anginosus (milleri) y

Hospital Padre Alberto Hurtado: Unidad de Laboratorio Clínico (PPM, PAL), Unidad de Gestión Clínica del Niño (JBO, PPD). 


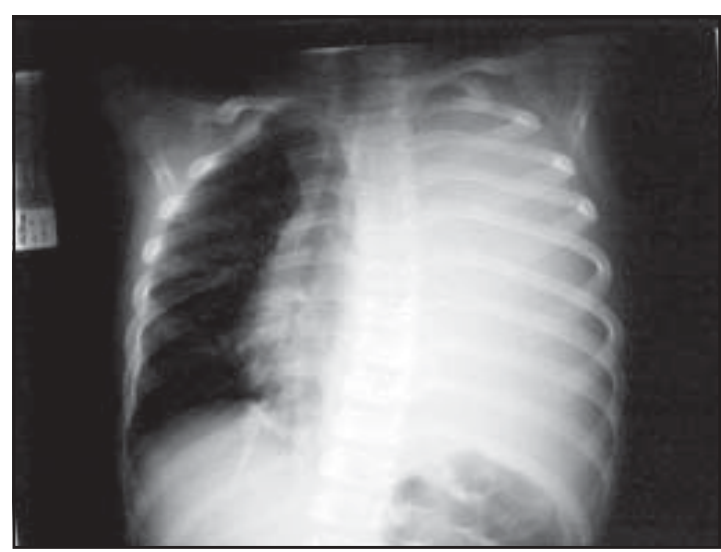

Figura 1. Radiografía de tórax que muestra colección intratorácica izquierda extensa, 43 días después de neumonectomía izquierda.

Prevotella spp, respectivamente. Se modificó entonces el esquema antimicrobiano a penicilina sódica más clindamicina, el que se prolongó durante 34 días. El drenaje pleural se mantuvo por 28 días, dando salida a 200 a $400 \mathrm{ml} /$ día los siete primeros días y luego $5 \mathrm{a} 10 \mathrm{ml} /$ día hasta el día 27. Fue dado de alta tras 39 días de hospitalización, en buenas condiciones. Evolucionó asintomático hasta su último control en diciembre del 2003. La evaluación genética descartó una genopatía y concluyó que la malformación pulmonar era de causa desconocida.

\section{Discusión}

Streptococcus grupo anginosus son cocáceas grampositivas en cadena, catalasa negativa, pertenecientes al género Streptococcus. En el Bergey's Manual of Systematic Bacteriology de 1984 se enunciaban sólo 7 géneros de cocáceas grampositivas anaeróbicas facultativas; en la actualidad se reconocen aproximadamente 20 géneros. En esta comunicación nos referiremos a un grupo bacteriano dentro de uno de estos géneros ${ }^{1}$.

Los esquemas clásicos de clasificación de Streptococcus incluyen principalmente la reacción hemolítica en agar sangre de cordero al 5\% y además, en aislados $\beta$ hemolíticos, la presencia de polisacárido en su membrana externa (grupo antigénico de Lancefield). A pesar de que con el desarrollo de la biología molecular se ha observado que cepas $\beta$ hemolíticas no relacionadas como especie, pueden producir idéntico antígeno de Lancefield, estos dos criterios continúan siendo útiles para dividir el género Streptococcus en amplias categorías, como un primer paso en la identificación de aislados clínicos ${ }^{1}$.

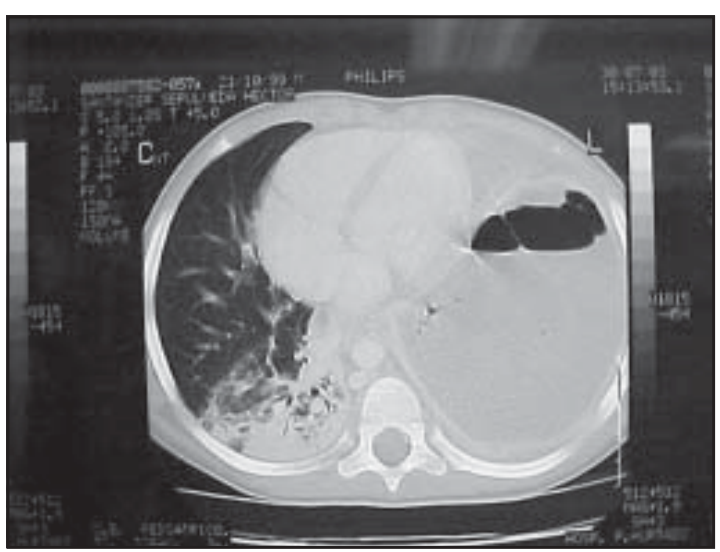

Figura 2. TAC pulmonar en el que se observa gran colección intrapleural izquierda que ocupa toda la cavidad y consolidación pulmonar basal derecha.

Con respecto a la relación genética entre las especies de Streptococcus, Kawamura et al, utilizando comparación de secuencias de ARNr de la subunidad $16 \mathrm{~S}$ para la caracterización del género Streptococcus, demostró la existencia del grupo piogénico y grupo no piogénico; este último incluye los grupos mitis, anginosus, mutans, salivarius y bovis. Este estudio además, demuestra una estrecha relación filogenética entre Streptococcus pneumoniae y especies del grupo mitis, sugiriendo la inclusión de este agente dentro de este último grupo ${ }^{1 \mathrm{a}}$.

En la Tabla 1 se describen las especies de Streptococcus $\beta$ hemolíticos clínicamente relevantes y su correspondiente agrupación, según antígeno de Lancefield. En esta tabla podemos observar que Streptococcus pyogenes (A) y Streptococcus dysgalactiae (C o G) poseen idéntico antígeno de Lancefield que algunas cepas de Streptococcus grupo anginosus, razón por lo cual, esta prueba debe complementarse con otras pruebas fisiológicas descritas en dicha tabla, para el correcto diagnóstico de laboratorio de especies de Streptococcus $\beta$ hemolíticos ${ }^{1,2}$.

Entre los Streptococcus no $\beta$ hemolíticos más frecuentemente relacionados con infecciones clínicas, se encuentran S. pneumoniae, Streptococcus grupo bovis ( $S$. bovis es la especie más conocida) y otros Streptococcus del grupo viridans descritos en la Tabla $2^{1,2}$.

Existe una gran confusión en la terminología y clasificación de Streptococcus grupo anginosus; en la actualidad no existe duda que es un grupo que posee 3 especies: $S$. anginosus, $S$. constellatus y S. intermedius, que fueron colectivamente conocidos como "Streptococcus anginosus" y "Streptococcus milleri”. El término "Streptococcus milleri" nunca fue aceptado por los taxonomistas 
Tabla 1. Especies y pruebas de laboratorio para la diferenciación de Streptococcus $\beta$ hemolíticos clínicamente relevantes

\begin{tabular}{|c|c|c|c|c|c|c|}
\hline $\begin{array}{l}\text { Grupo } \\
\text { Lancefield }\end{array}$ & $\begin{array}{l}\text { Tamaño } \\
\text { colonias }{ }^{1}\end{array}$ & Especie & PYR & $\mathbf{V P}^{2}$ & CAMP & BGUR \\
\hline A & Grande & S. pyogenes & + & - & - & ND \\
\hline A & Pequeña & S. grupo anginosus & - & + & - & ND \\
\hline B & Grande & S. agalactiae & - & & + & ND \\
\hline $\mathrm{C}$ & Grande & S. dysgalactiae & - & - & - & + \\
\hline $\mathrm{C}$ & Pequeña & S. grupo anginosus & - & + & - & - \\
\hline $\mathrm{F}$ & Pequeña & S. grupo anginosus & - & + & - & ND \\
\hline G & Grande & S. dysgalactiae & - & - & - & + \\
\hline G & Pequeña & S. grupo anginosus & - & + & - & - \\
\hline No agrupable & Pequeña & S. grupo anginosus & - & + & - & ND \\
\hline
\end{tabular}

${ }^{1}$ Tamaño de colonias: grande > de $5 \mathrm{~mm}$ de diámetro después de 24 horas de incubación

${ }^{2}$ Resultado del test VP para $S$. agalactiae ha sido omitido por diferentes resultados en la literatura.

+ : resultado del test positivo. -: resultado del test negativo. ND: Datos no disponibles. VP: Voges-Proskauer. PYR: pirrolidonil aminopeptidasa. CAMP: test de CAMP. BGUR: $\beta$ D- glucuronidasa

Tabla 2. Especies de Streptococcus grupo viridans

\begin{tabular}{ll}
\hline Grupo & Especies \\
\hline Grupo mitis & S. mitis, S. sanguis; S. parasanguis, S. gordonii, S. crista, S. oralis, S. pneumoniae \\
Grupo mutans & S. mutans, S. sobrinus, S. criceti, S. rattus \\
Grupo salivarius & S. salivarius, S. vestibularis, S. thermophilus \\
Grupo bovis & S. bovis, S. alactolyticus, S. equinus \\
Grupo anginosus & S. anginosus, S. constellatus, S. intermedius \\
\hline
\end{tabular}

como una confirmada entidad taxonómica; sin embargo, en la literatura médica, especialmente europea, este término se sigue empleando para referirse a este grupo bacteriano. Para mayor confusión, existen cepas $\beta$ hemolíticas y otras $\alpha$ hemolíticas o no hemolíticas, clasificadas estas últimas dentro del grupo viridans ${ }^{1-4}$.

Streptococcus grupo anginosus constituye parte de la flora residente de la orofaringe, nasofaringe, surcos gingivales, tracto gastrointestinal y vagina, y desde estos territorios migra a diversos sitios donde produce las infecciones ${ }^{1,5-7}$.

Poco se conoce acerca de los factores de patogenicidad de este grupo bacteriano; entre los factores de virulencia que promueven la colonización e invasión se describen una adhesina no fibrilar que genera unión a fibronectina en la célula huésped, inhibición de la actividad bactericida de neutrófilos y una citotoxina que además de generar destrucción de la célula huésped, contribuye a la sobrevida y persistencia bacteriana, por reducción de los PMN funcionantes.
Dentro de los factores de patogenicidad que dañan directamente al huésped, se ha demostrado que $S$. intermedius produce un conjunto de enzimas hidrolíticas que ayudan a la licuefacción del tejido y formación de abscesos, además de la citotoxina mencionada anteriormente. In vivo e in vitro existe evidencia de sinergismo con bacterias anaerobias como Fusobacterium nucleatum y Prevotella intermedia por supresión sinérgica de la actividad bactericida de PMN. Los factores de patogenicidad expuestos explican la naturaleza de las infecciones causadas por Streptococcus grupo anginosus que tienen tres características primordiales: son de naturaleza polimicrobiana, están implicados en la generación de abscesos y existe dificultad para su erradicación microbiológica ${ }^{8-11}$.

Streptococcus grupo anginosus ha sido reportado como causa de infecciones supurativas en niños y adultos. Sin embargo, en pediatría existen escasos reportes con un número significativo de casos. Belko et al realizaron un estudio clínicomicrobiológico retrospectivo en pacientes pediá- 
tricos en un hospital terciario de Boston. Revisaron los registros de laboratorio correspondientes a un período de 6 años obteniendo 213 aislados de Streptococcus grupo anginosus en 188 pacientes. Este estudio reveló que $76 \%$ de la infecciones por Streptococcus grupo anginosus eran de carácter polimicrobiano y en asociación principalmente con bacilos gramnegativos y anaerobios. El 18\% de los pacientes presentó factores de riesgo, entre los más frecuentes se encontraban procedimientos dentales, anormalidades congénitas y trauma abdominal. Streptococcus grupo anginosus fue aislado en $29 \%$ de las muestras de abscesos del SNC, en $20 \%$ de muestras de abscesos dentales, en $19,5 \%$ de muestras provenientes de infecciones intraabdominales, en $14 \%$ de abscesos peritonsilares, en 7,3\% de empiemas y abscesos pulmonares, en 1,4\% de las endocarditis infecciosas y en $0,1 \%$ de las bacteriemias en el periodo estudiado. En esta serie de casos no se observó mortalidad y de los 6 pacientes que presentaron infección pulmonar, la duración promedio del tratamiento antimicrobiano fue de 42 días, lo que concuerda con estudios que revelan dificultad de erradicación bacteriana y la necesidad de tratamientos prolongados ${ }^{12-15}$.

De las especies de Streptococcus grupo anginosus, se ha observado que $S$. intermedius es más frecuentemente aislado en infecciones del SNC, S. constellatus en infecciones pulmonares y $S$. anginosus en bacteriemias, infecciones intestinales y genitales ${ }^{16}$.

El diagnóstico de laboratorio no ha estado exento de dificultad. En las cepas $\beta$ hemolíticas existe un porcentaje que posee idéntico antígeno de Lancefield que $S$. pyogenes y $S$. dysgalactiae, por lo que utilizar sólo esta prueba para la diferenciación ha llevado a confusión. Por otro lado, en cepas no $\beta$ hemolíticas (hemólisis $\alpha$ o $\gamma$ ), no siempre se realiza, en la labor asistencial, la diferenciación de Streptococcus grupo anginosus den- tro del grupo viridans. Para un adecuado diagnóstico bacteriológico de Streptococcus grupo anginosus se deben considerar las características microbiológicas generales: cocáceas grampositivas en cadena, generan microcolonias (diámetro $<0,5 \mathrm{~mm}$ ), entre 7 y $38 \%$ de las cepas son $\beta$ hemolíticas (dependiendo de la especie aislada), el resto pueden ser $\alpha$ o no hemolíticas y poseen un característico olor acaramelado debido a la producción de diacetilo. A estas características se debe adicionar otras pruebas de laboratorio específicas según el tipo de hemólisis que presenten. En cepas $\beta$ hemolíticas, se recomienda las pruebas de laboratorio y diferenciación que se muestra en la Tabla 1, recordando que un tercio posee grupo antigénico de Lancefield (A, C, F o $\mathrm{G})$. En cepas no $\beta$ hemolíticas primero se recomienda descartar S. pneumoniae y Streptococcus bovis (especie clínicamente relevante dentro del grupo bovis) con las pruebas descritas en la Tabla 3, y posteriormente determinar si corresponde a Streptococcus grupo anginosus, para lo cual, se recomienda realizar a lo menos dos pruebas adicionales: Voges-Proskauer e hidrólisis de arginina, que resultan positivas en estas cepas $^{2,17-21}$. Los tests fenotípicos para la caracterización de especies dentro de este grupo bacteriano están basados principalmente en la actividad de distintas enzimas y su realización es bastante laboriosa para ser utilizada rutinariamente; con métodos semiautomatizados se ha observado 57 a $98 \%$ de concordancia con estudios genotípicos, lo que varía dependiendo de la especie aislada ${ }^{1,22-24}$.

Es importante mencionar algunos puntos sobre el informe de laboratorio. Con los antecedentes antes mencionado, en cepas $\beta$ hemolíticas parece lo más adecuado informar el nombre de la especie como: Streptococcus pyogenes, Streptococcus dysgalactiae o grupo bacteriano en el caso de Streptococcus grupo anginosus, y no informar sólo el grupo según resultado de la

Tabla 3. Pruebas de laboratorio para la diferenciación de Streptococcus no $\beta$ hemolíticos ${ }^{1}$

\begin{tabular}{lccc}
\hline & $\begin{array}{c}\text { Susceptibilidad } \\
\text { a optoquina }\end{array}$ & $\begin{array}{c}\text { Solubilidad en bilis } \\
\text { esculina }\end{array}$ & $\begin{array}{c}\text { Crecimiento en bilis } \\
\text { end }\end{array}$ \\
\hline S. pneumoniae & + & + & - \\
S. bovis & - & - & - \\
Otros Streptococcus grupo viridans & - & & ${ }^{3}$ \\
\hline$+:$ resultado del test positivo. & & \\
-: resultado del test negativo. \\
${ }^{1}$ Cepas de $S$. agalactiae pueden ser no $\beta$ hemolíticas, si existe la sospecha, debe realizarse pruebas adicionales. \\
${ }^{2}$ Algunas cepas de S. pneumoniae pueden dar resultados negativos de optoquina y solubilidad en bilis. \\
${ }^{3}$ Algunas cepas de otros Streptococcus grupo viridans pueden dar reacciones positivas, debe complementarse con \\
pruebas adicionales. Útil la aglutinación con antisuero grupo D que puede resultar (+) para S. bovis.
\end{tabular}


reacción serológica (Streptococcus $\beta$ hemolítico grupo A, C o G). En el caso de Streptococcus $\beta$ hemolítico grupo F y B se recomienda, siguiendo esta metódica, informarlo como Streptococcus grupo anginosus y Streptococcus agalactiae respectivamente, a pesar de que en estos casos puede utilizarse ambas formas, debido a que no existe otro grupo bacteriano que posea estos antígenos.

Con respecto a la susceptibilidad antimicrobiana se debe determinar la CIM y utilizar los criterios de interpretación de la NCCLS para Streptococcus grupo viridans (en cepas $\beta$ hemolíticas y no hemolíticas). La mayoría de los reportes demuestran buena actividad in vitro de penicilina y cefalosporinas de tercera generación para este grupo; sin embargo, existen reportes aislados que sugieren la emergencia de resistencia a penicilina, la que alcanza hasta $33 \%$ en $S$. intermedius. La resistencia a eritromicina varía entre 0 y $15 \%{ }^{12,25-30}$. Al momento de enviar este manuscrito, no existen publicaciones nacionales con respecto a susceptibilidad antimicrobiana en cepas de Streptococcus grupo anginosus, creemos que sería interesante realizar dicho estudio para conocer el comportamiento de cepas aisladas en nuestro país.

\section{Resumen}

Presentamos el caso clínico de un preescolar con antecedente de malformación pulmonar que 43 días tras una neumonectomía izquierda, ingresó al Hospital Padre Hurtado con fiebre de una semana de evolución. En una TAC pulmonar se demostró una gran colección intrapleural izquierda de la que se aisló Streptococcus grupo anginosus y Prevotella spp. Se efectuó tratamiento antimicrobiano con penicilina más clindamicina. Existe gran confusión en la terminología y clasificación de Streptococcus grupo anginosus. En la actualidad no existe duda que es un grupo que posee tres especies, $S$. anginosus, $S$. constellatus y $S$. intermedius. Son parte de la flora normal de orofaringe, nasofaringe, tracto gastrointestinal y vagina. Este grupo ha sido reconocido como causa de infecciones supurativas en niños y adultos. La mayoría de las cepas se describen como susceptibles a penicilina; sin embargo, existen reportes que sugieren la emergencia de resistencia. Se discuten las recomendaciones actuales para el diagnóstico e informe microbiológicos.

\section{Bibliografía}

1.- Facklam R. What happened to the Streptococci: Overview of taxonomic and nomenclature changes. Clin Microbiol Rev 2002; 15: 613-30.

1a.- Kawamura Y, Hou X G, Sultana F, Miura H, Ezaki T.
Determination of $16 \mathrm{~S}$ rRNA sequences of Streptococcus mitis and Streptococcus gordonii and phylogenetic relationships among members of genus Streptococcus. Int J Syst Bacteriol 1995; 45: 406-8.

2.- Ruoff K, Whiley R, Beighton D. Streptococcus. Murray P. Manual of Clinical Microbiology, $8^{\circ}$ Edition, Washington DC 2003: 405-21.

3.- Clarridge J, Osting C J, Jalali M, Osborne J, Waddington M. Genotypic and phenotypic characterization of Streptococcus milleri group isolates from a veterans administration hospital population. J Clin Microbiol 1999; 37: 3681-7.

4.- Teng L, Hsueh P, Tsai J, Chen P, Hsu J, Lai H et al. gro-ESL sequence determination, phylogenetic analysis, and species differentiation for viridans group Streptococci. J Clin Microbiol 2002; 40: 3172-8.

5.- Ahmet Z, Warren M, Houang E. Species identification of members of the Streptococcus milleri group isolated from the vagina by ID 32 Strep system and differential phenotypic characteristics. J Clin Microbiol 1995; 33: 1592-5.

6.- Turner J C, Fox A, Fox K, Addy C, Garrison C, Herron $\mathrm{B}$ et al. Role of group $\mathrm{C}$ beta-hemolytic Streptococci in pharyngitis: epidemiologic study of clinical features associated with isolation of group C Streptococci. J Clin Microbiol 1993; 31: 808-11.

7.- Fox K, Turner J, Fox A. Role of beta-hemolytic group C Streptococci in pharyngitis: incidence and biochemical characteristics of Streptococcus equisimilis and Streptococcus anginosus in patients and healthy controls. J Clin Microbiol 1993; 31: 804-7.

8.- Macey M, Whiley R, Miller L. Effect on polymorphonuclear cell funtion of a human-specific cytotoxin, intermedilysin, expressed by Streptococcus intermedius. Infect Immun 2001; 69: 6102-9.

9.- Nagamune H, Whiley R, Goto T, Inai Y, Maeda T, Hardie $\mathrm{J}$ et al. Distribution of intermedilysin gene among the anginosus group Streptococci and correlation between intermedilysin production and deep-seated infection with Streptococcus intermedius. J Clin Microbiol 2000; 38: 200-26.

10.- Shinzato T, Satio A. Mechanism of pathogenicity of Streptococcus milleri group in pulmonary infection: synergy with an anaerobe. J Med Microbiol 1994; 40: 118-23.

11.- Nagamune H, Ohnishi C, Katsuura A, Fushitani K, Whiley R, Tsuji A et al. Intermedilysin, a novel cytotoxin specific for human cells, secreted by Streptococcus intermedius UNS 46 isolated from a human liver abscess. Infect Immun 1996; 64: 3093 100.

12.- Belko J, Golmann D, Macone A. Clinically significant infections with organisms of the Streptococcus milleri group. Pediatr Infect Dis J 2002; 21: 715-26.

13.- Cade A, Denton C, Brownlee K, Todd N and Conway SP. Acute bronchopulmonary infection due to Streptococcus milleri in a child with cystic fibrosis. Arch Dis Child 1999; 80: 278-9.

14.- Jerng J, Hsueh P, Teng L, Lee L, Yang P, Luh K. Empyema thoracic and lung abscess caused by viridans Streptococci. Am J Respir Crit Care Med 1997; 156: 1508-14.

15.- Jacobs J, Tjhie J, Smeets M, Schot C, Schouls L. Genotyping by amplified fragment length polymorphism analysis reveal persistence and recurrence of infection with Streptococcus anginosus group organisms. J Clin Microbiol 2003; 41: 2862-6.

16.- Clarridge J, Attorri S, Musher D, Hebert J, Dunbar S. 
Streptococcus intermedius, Streptococcus constellatus, and Streptococcus anginosus (Streptococcus milleri group) are of different clinical importance and are not equally associated with abscess. Clin Infect Dis 2001; 32:1 511-5.

17.- Whiley R, Frase H, Hardif J, Beighton D. Phenotypic differentiation of Streptococcus intermedius, Streptococcus constellatus, and Streptococcus anginosus strains within the "Streptococcus milleri group". J Clin Microbiol 1990; 28: 1497-501.

18.- Chew T A, Smith J M. Detection of diacetyl (caramel odor) in presumptive identification of the Streptococcus milleri group. J Clin Microbiol 1992; 30: 3028-9.

19.- Rubin L, Kahn R, Vellozzi E, Isenberg H. False positive detection of group A Streptococcus antigen resulting from cross-reacting Streptococcus intermedius (Streptococcus milleri group). Pediatr Infect Dis J 1996; 15: 715-7.

20.- Johnson D, Kaplan E. False-positive rapid antigen detection test results: reduced specificity in the absence of group A Streptococci in the upper respiratory tract. J Infect Dis 2001; 183: 1135-7.

21.- Hamrick H, Mangum M. Beta- hemolytic Streptococcus milleri group misidentified as Streptococcus pyogenes on throat culture. Pediatr Infect Dis J 1999; 18: 75-6.

22.- Kirby R, Ruoff K. Cost-effective, clinically relevant method for rapid identification of beta-hemolytic Streptococci and Enterococci. J Clin Microbiol 1995; 33: 1154-7.

23.- Bascomb S, Manafi M. Use of enzyme test in characterization and identification of aerobic and facultatively anaerobic gram-positive cocci. Clin Microbiol Rev 1998; 11: 318-40.
24.- Kikuchi K, Eneri T, Totsuka K, Shimizu K. Comparison of phenotypic characteristics, DNA-DNA hybridization results, and results with a commercial rapid biochemical and enzymatic reaction system for identification of viridans group Streptococci. J Clin Microbiol 1995; 33: 1215-22.

25.- Bantar C, Fernández L, Relloso C, Lanza A, Bianchini $\mathrm{H}$ and Smayevsky J. Species belonging to the Streptococcus milleri group: antimicrobial susceptibility and comparative prevalence in significant clinical specimens. J Clin Microbiol 1996; 34: 2020-2.

26.- Doern G, Ferraro M, Brueggemann, Ruoff L. Emergence of high rates of antimicrobial resistance among viridans group Streptococci in United States. Antimicrob Agents Chemother 1996; 40: 891-4.

27.- Tracy M, Wanahita A, Shuhatovich Y, Goldsmith E, Clarridge J, Musher D. Antibiotic susceptibilities of genetically characterized Streptococcus milleri group strains. Antimicrob Agents Chemother 2001; 45: 1511-4.

28.- Aracil B, Gómez J, Alos J. A study susceptibility of 100 clinical isolates belonging to the Streptococcus milleri group to 16 cephalosporins. J Antimicrob Chemother 199; 43: 399-402.

29.- Jacobs J, Van Baar G, London N, Tjhie J, Schouls L, Stobbering H. Prevalence of macrolide resistance genes in clinical isolates of the Streptococcus anginosus ("S. milleri") group. Antimicrob Agents Chemother 2001; 45: 2375-7.

30.- National Committee for Clinical Laboratory 31. Standards (NCCLS). Performance standards for antimicrobial susceptibility testing. Thirteenth Informational Supplement. M 100-S 13(M7). 2003.

Correspondencia a:

Paola Pidal Méndez

E-mail: ciih@hurtadohosp.cl 